\title{
Individualised proteome profiling of human endometrial tumours improves detection of new prognostic markers
}

\author{
S Attarha ${ }^{1,2}$, S Andersson $^{2}, \mathrm{M} \mathrm{Mints}^{2}$ and S Souchelnytskyi ${ }^{\star, 1}$ \\ ${ }^{1}$ Department of Oncology-Pathology, Karolinska Institutet, Z5:01, KS, Solna, 17176 Stockholm, Sweden and ${ }^{2}$ Department of \\ Women's and Children's Health, Karolinska Institutet, KS, Solna, 17176 Stockholm, Sweden
}

Background: The individual features of tumours are often disregarded in cohort studies. As these features may represent a source for individualised cancer treatment, it is important to develop a novel approach for their assessment.

Methods: We used proteomics, systems biology, and immunohistochemistry to explore protein expression in human endometrial tumours, to identify deregulated regulatory mechanisms, and to validate observed changes in protein expression using tissue microarrays.

Results: Compared with the evaluation of common tumour features, the evaluation of individual tumour features gave a more comprehensive and detailed overview of the regulatory processes in endometrial tumours. Systemic analysis of the individual proteome profiles showed that endometrial tumours employed different proteins to regulate similar functions. Comparison of our data with publicly available data sets of molecular profiling of human endometrial tumours confirmed that individual tumour features are not simply irrelevant individual variations, but are indeed important in endometrial tumorigenesis. Validation through tissue microarray investigation of MST1 and PKN1 proteins confirmed the usefulness of this approach, and suggested that MST1 and PKN1 may be considered as predictive biomarkers of endometrial cancer.

Conclusion: We show that individualised profiling of endometrial tumours may deliver better insights into a tumour's physiology, thereby giving a better prediction of tumour development. Individual tumour features may also be used to tailor cancer treatment.

Molecular profiling of tumours is expected to improve cancer diagnostics and treatment. Potential biomarkers are often sought by analysing data that are common to a large number of patients, disregarding any changes that are specific to a single patient. However, as recent large-scale studies of human tumorigenesis have shown a significant variability in cancer-related changes on an individual level (Saunders et al, 2012; Tian et al, 2012), it is essential to explore whether the individual features of tumours are important in understanding tumorigenesis, diagnostics, and treatment selection.

Up to $10 \%$ of endometrial cancers develop into an aggressive form of the disease, with 5 -year survival rates between 5\% and $10 \%$ (Mhawech-Fauceglia et al, 2010). Histopathology is primarily used to differentiate between aggressive and non-aggressive cancers (Levan et al, 2010). However, histopathology does not reflect the molecular properties of tumours, which may explain why $>20 \%$ of endometrial cancers that are first assumed to be non-aggressive have been reported to develop into aggressive and metastatic cancer (Salvesen et al, 2009). The current limitations of histopathological diagnosis of endometrial cancer require the development of biomarkers that reflect the molecular functional profile of endometrial tumours.

Despite a number of efforts, there are currently no known molecular biomarkers that can predict the aggressiveness of endometrial cancer (Salvesen et al, 2012). Promising prognostic results have recently been reported for in situ biomarkers, such as 
DNA ploidy (Susini et al, 2007; Pradhan et al, 2012), P53 expression (Salvesen et al, 1999; Engelsen et al, 2006), and oestrogen and progesterone receptors (Kauppila et al, 1986; Creasman, 1993). Most of these studies have been retrospective, and were performed on patients who were not treated with stateof-the-art diagnostic imaging, histological subtyping, and lymphnode sampling (Salvesen et al, 2012). Recent comprehensive molecular profiling of primary tumours has identified the PI3K/ PTEN/AKT/mTOR pathway and FGFR2 as promising targets for development of drugs (Dedes et al, 2011; Salvesen et al, 2012; Westin and Broaddus, 2012). Therefore, these proteins may be used for companion diagnostics of the corresponding drugs.

Several proteomics-based studies of endometrial cancer have been published (Doll et al, 2008; Monge et al, 2009; Yi et al, 2009; Sharon et al, 2010; Attarha et al, 2011). A number of protein biomarkers have been associated with clinical characteristics and prognosis of endometrial cancer, but none are currently being used in routine clinical practice (Staff et al, 2011). In addition, these claimed markers do not represent known potent regulators of molecular processes, but rather high abundance proteins. These proteins are involved in proliferation, viability, and invasion of endometrial cancer cells (Yi et al, 2009), providing the migratory and invasive capabilities associated with the switch to myometrial infiltration (Monge et al, 2009), activating and repressing the transcription of key regulators of the growth, survival, and differentiation pathways (Doll et al, 2008).

High abundance proteins have been identified more frequently than regulatory proteins partly because there is significant variability between individual tumours. The traditional manner of identifying cancer-specific changes includes the generation of a list of expressed proteins that are common to all cancer cases, and a separate list of proteins that are common to cancer-free controls. These lists are then compared; the differences between them are expected to represent true cancer-specific changes. However, this approach assumes that cancer development follows the same path, and exhibits the same changes, in all cancer cases, whereas the heterogeneity of tumours suggests that this approach misses the individual features of tumours. Here, we report that previously neglected individual features of endometrial tumours provide a rich source of information about the molecular processes that are affected therein, and that the regulators of these processes may be used to predict the aggressiveness of endometrial cancer.

\section{MATERIALS AND METHODS}

Collection and preparation of endometrial epithelial tissue samples. Endometrial clinical samples were collected at the Department of Women's and Children's Health, Karolinska University Hospital (Stockholm, Sweden), under the Ethical Permit 2006/649. Samples were collected from women who underwent surgery; they were put on ice and sent to a pathologist for preparation, after which they were then frozen at $-70{ }^{\circ} \mathrm{C}$ for use in proteome profiling. Immunohistopathological diagnostics was performed at the Karolinska University Hospital. The samples used for the proteomics study were evaluated for histo-morphological features, for example, presence of different types of cells, stroma, and inflammatory cells.

Proteome profiling. For proteome profiling, samples were extracted directly into a rehydration buffer (8 $\mathrm{m}$ urea, $2 \%$ CHAPS, $0.002 \%$ Bromophenol blue, $0.28 \%$ Dithiothreitol, $0.5 \%$ ampholites 3-10 $\mathrm{pH}$ gradient (IPG) buffer), by mechanical disintegration with glass beads at room temperature. Tissue extracts were centrifuged at 13000 r.p.m. for $15 \mathrm{~min}$, and supernatants were used for twodimensional gel electrophoresis (2D-GE).
2D-GE, gel image analysis, and MALDI TOF mass spectrometry were performed as previously described (Zakharchenko et al, 2011). Briefly, first-dimension isoelectric focusing (IEF) was performed using $18 \mathrm{~cm}$ linear Immobiline Drystrips with a $\mathrm{pH}$ range of 3-10 in an IPGPhor instrument (GE Healthcare, Uppsala, Sweden) by passive rehydration for $10 \mathrm{~h}$ at $20^{\circ} \mathrm{C}$ and following electrophoresis $(50 \mathrm{~V}$ for $3 \mathrm{~h}, 1000 \mathrm{~V}$ for $1 \mathrm{~h}$, and $5000 \mathrm{~V}$ for $10 \mathrm{~h}$ or until reaching $32000 \mathrm{Vhr}$ ). Before second-dimension SDS-PAGE, the IPG strips were equilibrated in two steps with equilibration buffer (50 mu Tris-HCl, pH 8.8, $6 \mathrm{~m}$ urea, 30\% (v/v) glycerol, $2 \%$ $(\mathrm{w} / \mathrm{v})$ SDS, $0.002 \%(\mathrm{w} / \mathrm{v})$ Bromophenol blue). Samples were alkylated with iodoacetamide. Second-dimension SDS-PAGE was performed in an Ettan Dalt Six (GE Healthcare). At least two good quality $12 \%$ SDS-PAGE gels were generated for each sample. Generated gels were stained with silver nitrate. Protein spots were analysed using dedicated software (Image Master Platinum v6.0; GE Healthcare). Statistical significance of the reproducibility of protein spot expression in $2 \mathrm{D}$ gels, and of the differences in protein expression, was evaluated using the tools embedded in Image Master 2D platinum software. Student's $t$-test was used to ensure statistical significance of protein spot selection $(P<0.05)$. For each individual case, proteins that showed either a more than two-fold change in their expression pattern, or that were unique for a given tumour or to a corresponding histologically normal adjacent tissue were selected for identification by mass spectrometry.

Protein identification. Protein spots were excised from the gels, destained, and subjected to in-gel digestion with trypsin (modified sequence-grade, porcine; Promega, Madison, WI, USA). Tryptic peptides were concentrated and desalted on a $\mu$ C18 ZipTip (Millipore, Billerica, MA, USA). Peptides were directly eluted with $50 \%$ acetonitrile containing $\alpha$-Cyano- 4 -hydroxycinnamic acid matrix onto the metal target and analysed by MALDI TOF MS on Micromass' M@LDI-Reflectron instrument (Waters Corp., Milford, MA, USA). Embedded Micromass software (MassLynx Software v4.0; Waters Corp.) was used to process the mass spectra. Peptide spectra were internally calibrated using autolytic peptides from the trypsin $(842.510,1045.564$, and $2211.105 \mathrm{Da})$. To identify proteins, we performed searches in the NCBI's nr sequence database using the ProFound search engine (http://65.219.84.5/service/prowl/profounf.html). One missed cleavage, alkylation with iodoacetamide and partial oxidation of methionine were allowed. Search parameters were set on mass tolerance $<0.1, \mathrm{pI}$ and $\mathrm{Mr}$ as compared with the migration position of a spot in the $2 \mathrm{D}$ gel, and 'homo sapiens' was selected for species search. Significance of the identification was evaluated according to the probability value, $Z$-value, mass precision of the matched peptides and sequence coverage.

Systemic analysis. Protein names were translated into Gene Ontology terms (http://biodbnet.abcc.ncifcrf.gov/). Systemic analysis of obtained data was performed using GoMiner (http://discover. nci.nih.gov/gominer/) and Cytoscape tools. GoMiner provides a classification of identified proteins into biologically coherent categories, and assesses these categories. Relationships between identified proteins were explored by Cytoscape, and a network of identified proteins was generated for analysis. MiMIplugin was used to extract relevant proteins and RNAs from public databases. The network was viewed in Cytoscape; betweenness was computed by Network analysis tool, and network modules were extracted by AllegroMCODE tool. Fisher's exact test was used to calculate the $P$-value determining the network connectivity.

The FunCoup tool (www.funcoup.sbc.su.se) was used to build the small scale networks. Confidence score threshold was set at 0.50 , to ensure significance of the network.

Immunohistochemistry. UT501 USBiomax (USBiomax Inc., Rockville, MD, USA) and EMC1021 (Pantomics Inc., Richmond, CA, USA) endometrial cancer arrays were used to evaluate the 
expression of PKN1 and MST1. The UT501 array slide contained 41 cases of endometrioid adenocarcinoma, 2 cases of serous adenocarcinoma, 2 cases of clear cell adenocarcinoma, and 5 normal tissues, while the EMC1021 array slide contained 5 normal tissues and 97 cases of endometrial cancer. Arrays were stained with anti-PKN1 (H-234, Santa Cruz Biotechnology Inc., Santa Cruz, CA, USA) and anti-MST1 (AP7922a; Abgent Inc., San Diego, CA, USA) at a dilution of $1: 50$ according to the supplier's recommendations. Antigen retrieval was performed using DakoCytomation target retrieval solution high $\mathrm{pH}$ (DAKO, Carpinteria, CA, USA). The slides were stained with VECTASTAIN Elite ABC kits (Vector Laboratories Inc., Burlingame, CA, USA) following the manufacturer's instructions, counterstained with haematoxylin and mounted with Fluoromount G (Southern Biotechnology, Birmingham, AL, USA). The stained tissues were photographed using a Leica DFC camera and images were acquired with Leica QWin Standard software (Leica Microsystems Imaging Solutions Ltd, Cambridge, UK). Intensity of staining was evaluated as absent $(-)$, weak staining in fewer than $5 \%$ of cells $(+)$, staining in $>5 \%$ but $<50 \%$ of cells $(++)$, moderate staining in $>50 \%$ of cells $(+++)$ and strong staining in $>50 \%$ of cells $(++++)$. Staining was evaluated in malignant (epithelial) cells of tumours and epithelial cells of normal tissues. Sensitivity and specificity for MST1 and PKN1 expression in tumours and normal tissues were calculated. Fisher's exact test was used to calculate the significance of differences in expression of MST1 and PKN1 in IHC study.

\section{RESULTS}

Proteome profiling of individual tumours. We applied a twostep strategy to analyse the contribution of individual features of tumours to endometrial tumorigenesis. The first step was proteome profiling of three individual cases. The second step was a metaanalysis of individual features of endometrial tumours, which was used as a source to identify possible biomarkers, and included immunohistochemical validation within a separate set of endometrial cancer cases.

Histological analysis of the three cases used for proteome profiling showed typical histology for endometrial cancer (Figure 1A and B). All three were diagnosed as stage IA type 1 endometrioid endometrial carcinomas (EECs), with histologically detected invasion into the myometrium. Histological analysis showed no significant differences between the cases in representation of cells and stroma. Therefore, the differences in protein expression due to the morphological differences were minimised. However, as such intertumour differences cannot be fully excluded, we performed IHC study to validate expression pattern of selected identified proteins. As we explored the importance of the individual features of tumours, a limited number of cases were used for the discovery part of this work. For the validation study of markers selected from our proteomics data, we tested tissue microarrays containing total 168 endometrial cancer and non-cancer/normal cases.

We studied intact proteins, which is more informative due to detection of the proteins as they are in tumour samples, instead of artificially generated peptides, as in the peptide-based approaches by MS/MS. We used 2D gel electrophoresis, which allows for the detection of $>2000$ intact proteins and their isoforms in a single run. Proteome maps were generated for the three EC cases of tumour samples, and for histologically normal adjacent tissues for cases \#2 and \#3 (Figure 1C; Supplementary Figure S1). Normal adjacent tissue was not available for case \#1. The overall patterns of protein migration in $2 \mathrm{D}$ gels were similar for all three cases. This indicates that the isoelectric points and molecular masses of studied proteins did not differ between the studied cases. It also indicates that the overall morphological structures of the studied samples were similar, for example, presence of malignant cells and stromal components. The pattern similarity reflects the same histological origin (Supplementary Figure S1). All identified proteins are given in Supplementary Table S1.

Despite similarity of the overall separation pattern of the proteins, we observed significant variability between the three tumours in identities of tumour-related proteins. In all, 298 tumour-related protein spots were detected for case \#1, while for cases \#2 and \#3 there were 121 and 165 tumour-related protein spots, respectively. The lists of proteins identified in each case are given in Supplementary Table S1. Analysis of these lists showed high variability in identities of the tumour proteins across the three cases. To explore what this variability may reflect, we performed molecular profiling of the tumours, using the proteins identified through proteome profiling.

Molecular profiling. The proteins identified through proteome profiling represented two types of features - those common to many endometrial cancers and those specific to a single tumour. To explore whether any of the individual variability observed was relevant to tumorigenesis, we generated separate molecular profiles for each case. We assumed that although the individual tumour features we observed differed between cases, they may have led to the same functional output, and that discarding these individual differences and processing only common features may lead to the loss of important information on the regulatory profiles of these tumours.

Networks were built for each EEC case to characterise the molecular profiles of the tumours, using the proteins identified through proteome profiling (Supplementary Table S1; Supplementary Figure S3). These networks were used to identify which signalling mechanisms were affected in the tumours. It has been well established that key cellular functions may be regulated in the same manner by different components of a given signalling network (Souchelnytskyi, 2005). For example, cell proliferation can be affected by $>100$ proteins, including cyclins, CDK, CDK inhibitors, growth factors, MAP kinase pathways, and various tyrosine and serine/threonine kinases and phosphatases. Our hypothesis is that different proteins may be affected in the tumours of different individuals, but that these proteins may have a similar impact on the fundamental cellular functions. Therefore, the hypothesis of 'many means to the same end' was tested by analysis of identified proteins on a case-by-case basis, followed by a meta-analysis of individual data sets to find common functional mechanisms.

First, we established which functional domains were affected by the proteins identified in a given tumour. Then, these case-related functional domains were compared across the three EEC cases (Figure 2). As expected, we detected a significant overlap of functional domains between the three EEC cases. The overlapping high-level domains represent regulation of cell growth and proliferation, cell migration, immune response regulation, response to hormones, and angiogenesis regulation (Figure $2 \mathrm{~A}$ ). This overlap indicates that the affected mechanisms in the tumours are of similar general output. However, when we analysed the components of the individual networks, we observed no overlap (Figure $2 \mathrm{~B}$ ). This finding indicates that the tumours are using different mechanisms to achieve similar goals, for example, uncontrolled growth. The importance of this observation is related to treatment selection. Different individual features of a tumour may be targeted by different drugs (Figure 2C). For example, methotrexate may be helpful for case \#1, salinomycin for case \#2, and trichostatin A for case \#3. This demonstrates how the consideration of individual tumour features provides a rich source for a truly individualised treatment design.

Systemic analysis of invasiveness-associated proteome signature. Systemic analysis of the networks of identified proteins created for the three EEC cases showed that the variability in identified 


\begin{tabular}{|c|c|c|c|c|c|c|}
\hline $\begin{array}{c}\text { Case } \\
\text { number }\end{array}$ & Age & Type & Stage & Grade & $\begin{array}{c}\text { ER and } \\
\text { PR status }\end{array}$ & $\begin{array}{c}\text { p53/p21 } \\
\text { expression }\end{array}$ \\
\hline$\# 1$ & 63 & 1 & IA & 3 & $\begin{array}{c}\text { ER40\% } \\
\text { PR80\% }\end{array}$ & 0 \\
\hline$\# 2$ & 88 & 1 & IA & 2 & $\begin{array}{c}\text { ER80\% } \\
\text { PR80\% }\end{array}$ & + \\
\hline$\# 3$ & 75 & 1 & IA & 1 & $\begin{array}{c}\text { ER100\% } \\
\text { PR80\% }\end{array}$ & 0 \\
\hline
\end{tabular}
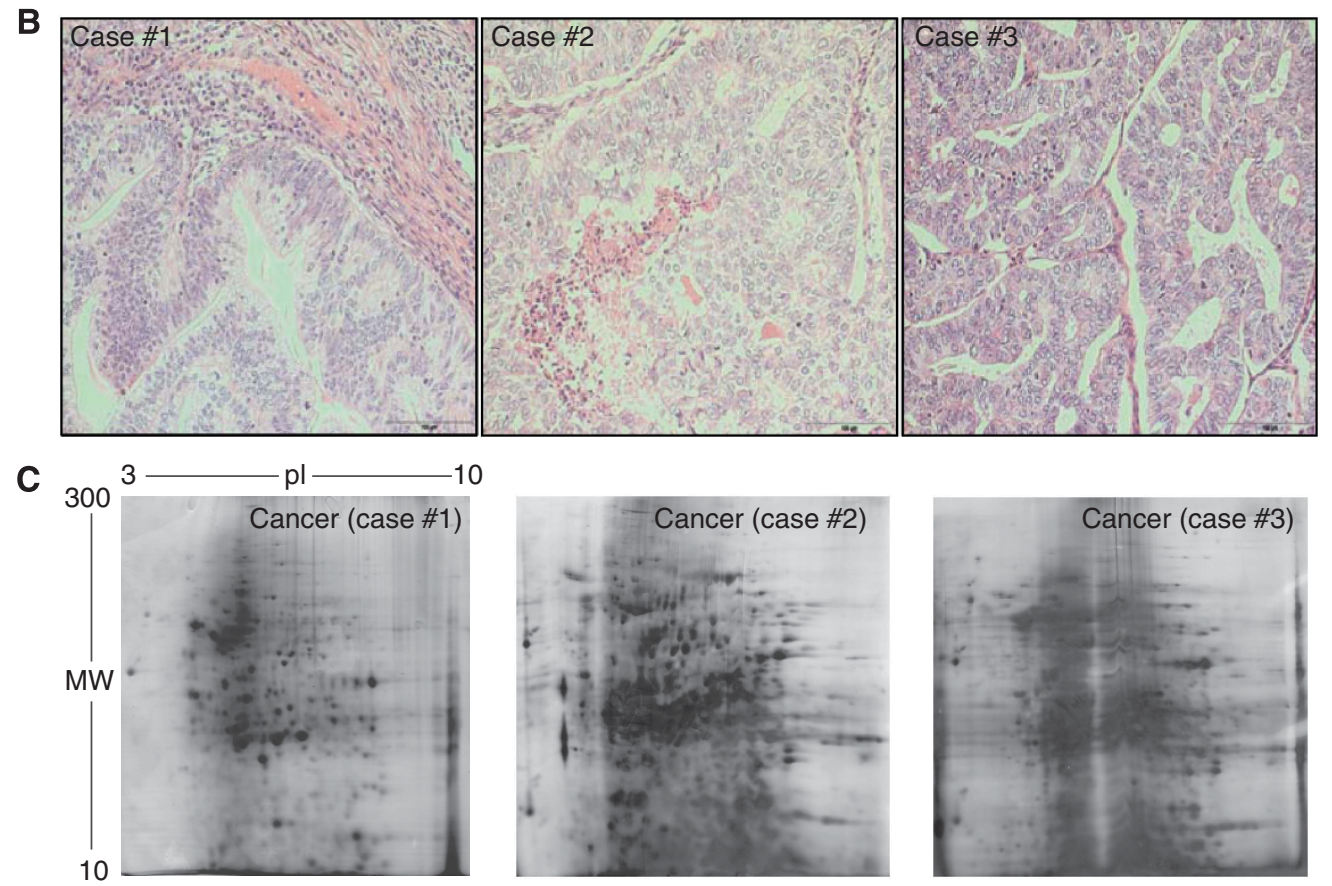

Figure 1. Proteome profiling. (A) Description of the three tumours subjected to proteome profiling. (B) Histology of the tumours of the three EEC cases subjected to the proteome profiling is shown. (C) Images of representative 2D gels. The images show the separation of the proteins extracted from tumours. Directions of isoelectric focusing and SDS-PAGE are indicated on the top and on the side of the left gel image, and are the same for the two other images. Gel images for each EEC case are shown in Supplementary Figure S1. A list of identified proteins is given in Supplementary Table S1.

proteins was translated into the involvement of similar functional domains (Figure 2; Supplementary Figure S3).

To assess the clinical value of observed individual features of tumours, an evaluation of a large number of cases is required. Therefore, we first explored publically available databases of clinical correlation studies, which are a rich source of results of clinical studies, and allow fast and reliable detection and validation of correlations of protein expression and clinical features, for example, survival or response to treatment. We used data sets deposited at the NCBI Gene Expression Omnibus and EBI to search for the components of the protein networks constructed from our three EEC cases (Supplementary Figure S4). The data sets we selected represent aggressive and non-aggressive endometrial cancer cases, separately.

To explore the systemic features of the signalling mechanisms represented by the proteins associated with invasive and noninvasive phenotype, networks of interactions were generated between these proteins and the proteins and genes they may affect, and the genes which were validated by analysis of published data sets (Figure 3; Supplementary Figures S3 and S4). We focused on invasiveness as it is the first step in the development of an aggressive cancer. This expansion of the network allowed to identify invasive-associated dependencies as a network with 441 nodes, while the network with non-invasive-associated dependencies had 616 nodes (Supplementary Figure S3G and H). Topology analysis showed that the both networks have features of a scale-free network. The nodes of high importance for robustness of a scalefree network are often the nodes of highest weight in the network, and highest centrality parameters such as betweenness (Bork et al, 2004). These nodes represent key regulators, as they have strongest impact on the system stability and response to perturbation. In the context of aggressiveness and non-aggressiveness, we expect that the key nodes of the invasive-associated and non-invasiveassociated networks would be crucial regulators of the molecular signature of aggressiveness. Analysis of the degree of connectivity of nodes identified TAF1, ACTA1, HNF4A, and ZBTB16 as potential key regulators for non-aggressive-specific network, while ATF2, JUN, TAF1, HNF4A, and ATF7IP identified as potential regulators for aggressive-specific network (Figure 3; Supplementary Figures S2 and S3). The impact of other nodes, for example, MYC, Max, SMAD3, TP53, and EGFR, was estimated to be significant due to higher betweenness, which is expected for a node with such connectivity. Network modules generated by the AllegroMCODE tool embedded in Cytoscape were selected from aggressive-specific and non-aggressive-specific networks (Supplementary Figure S3). The network modules showed a higher density of interconnections, 
as compared with the overall degree of connectivity in each network. These modules represented protein degradation regulation, cell apoptosis, cell proliferation, cell death, and cell-cycle regulation. Thus, the network analysis showed involvement in

A

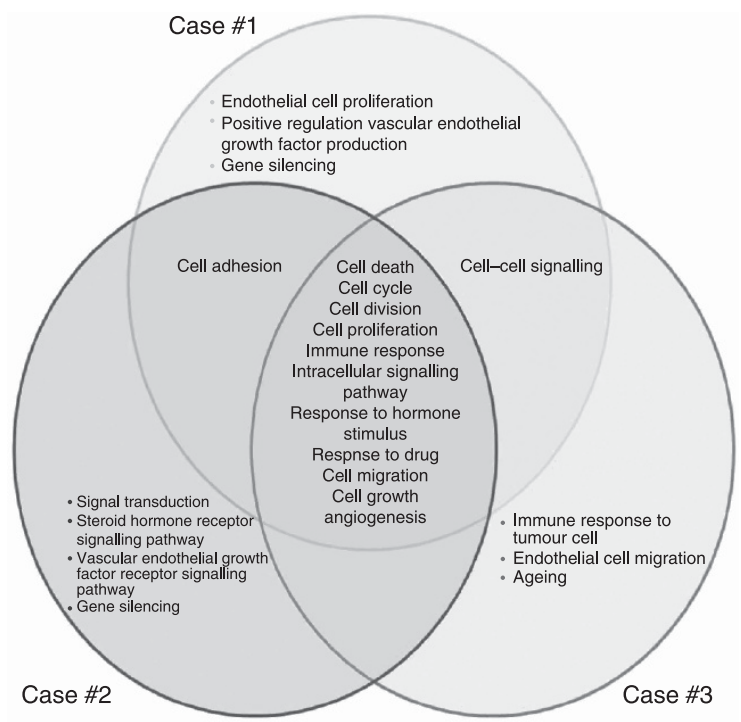

B

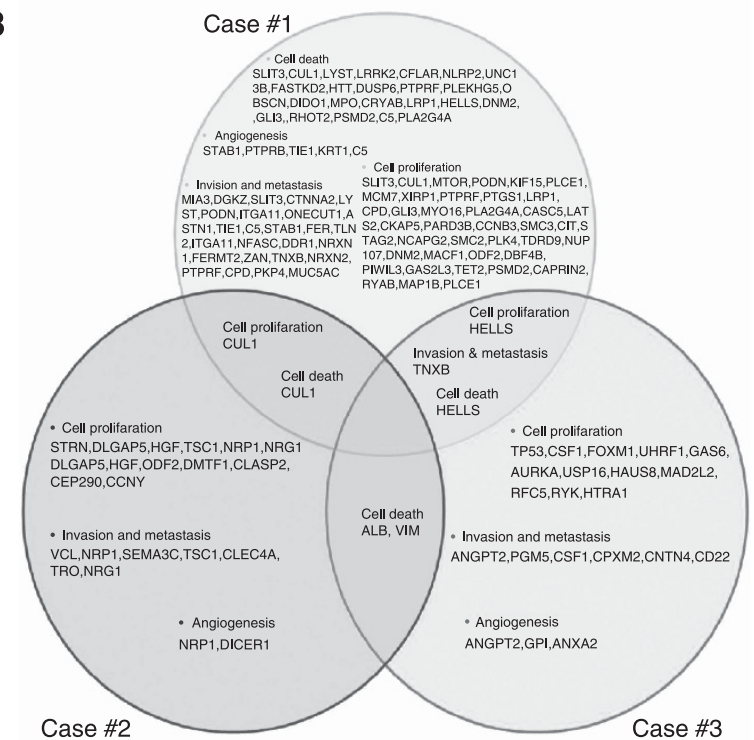

C

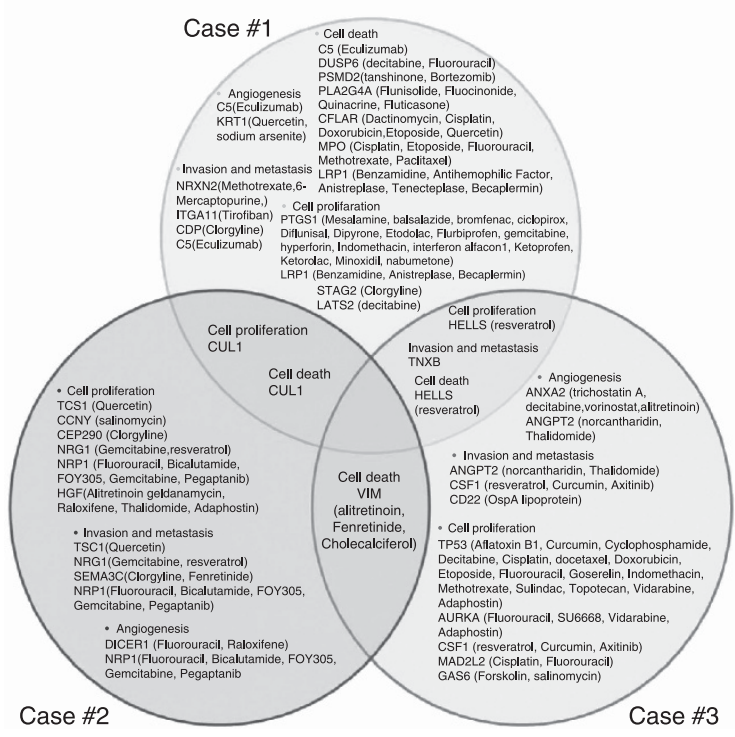

invasiveness of the endometrial cancer of many known regulators of tumorigenesis, such as MYC, TP53, EGFR, Jun, and Samd3. This analysis pointed also to the importance of regulators that have not been previously associated with endometrial cancer, such as ATF2, TAF1, UCP2, EXOSC10, and ATF7IP.

To validate the value of identified proteins and their networks as predictive biomarkers of the aggressiveness of a given tumour, we further explored aggressive and non-aggressive signatures by extracting dependencies that were common to the networks of proteins among cases, and for species in the data sets deposited at the ArrayExpression database of EBI (Figures 3 and 4; Supplementary Figure S4). Despite relatively limited overlap in the lists of genes and proteins in the studied data sets, we observed a significant correlation between upregulated genes and proteins for non-aggressive (10 proteins and genes) and aggressive (7 proteins and genes) signatures. We also observed that these proteins may initiate networks that regulate a number of functions. These functions are illustrated as subnetworks in Figure 4. The observed correlation between proteome profiling and gene expression data is very important, as it confirms that our proteome profiling of few cases detected changes that were also observed in our large-scale study. This provides confidence and supports the further study of the proteins identified in this study as markers of invasiveness and aggressiveness of endometrial cancer.

PKN1 and MST1 are downregulated in aggressive endometrial cancer. PKN1 and MST1 were selected for validation by tissue microarrays. These proteins were selected due to reports of their potential role in endometrial cancer (Galgano et al, 2009; $\mathrm{Ng}$ et al, 2013). We performed a validation study on the same three cases that were used for proteome profiling, plus additional cases of endometrial cancer, and tissue microarray of endometrial cancer specimens (Figure 5; Supplementary Figure S5). In all, 168 cases were analysed in two different tissue microarrays. We observed an increase in the expression of MST1 in the normal endometrium compared with tumour tissues (Figure 5A and B; Supplementary Figure S5). UT501 tissue microarrays showed strong MST1 staining $(++++)$ exclusively in normal endometrium, and EMC1021 tissue microarrays samples showed slightly weaker staining $(+++)$, but in the same high number of cells. Tumour cells consistently showed staining for MST1 in a lower number of cells and of weaker intensity. A similar observation was made for PKN1 staining (Figure 5C and D; Supplementary Figure S5). We did not observe significant differences in intracellular nuclear $v s$ cytoplasmic distribution of the staining signals. For both TMAs, UT501 and EMC1021, application of the Fisher's exact test showed that the differences in expression of MST1 and PKN1 between normal and malignant cells were significant, with $P$-values of $<0.01$ for all four arrays and studies (see legend to Supplementary Figure S5C for details). Sensitivity of immunohistochemical staining for MST1 was 98\% (UT501) and 72\% (EMC1021), and specificity was 100\% (UT501) and $80 \%$ (EMC1021) in tissue

Figure 2. Individualisation of data analysis leads to increased insights into affected functions. The Venn diagrams show (A) overlaps between functional domains defined by the proteins identified in all three EEC cases and (B) overlaps between individual components in the functional domains mentioned in (A). Note the lack of overlap between all three EEC cases when individual components were considered, and the appearance of the overlap when functions affected by these proteins were compared. (C) Proteins specific to individual cases may indicate which drugs would be applicable to that specific patient. The diagrams were built upon analysis of the identified proteins using a GoMiner tool. 'Cellular functions' category was selected for the analysis of affected functions. 
A Centiscape scatter plot view
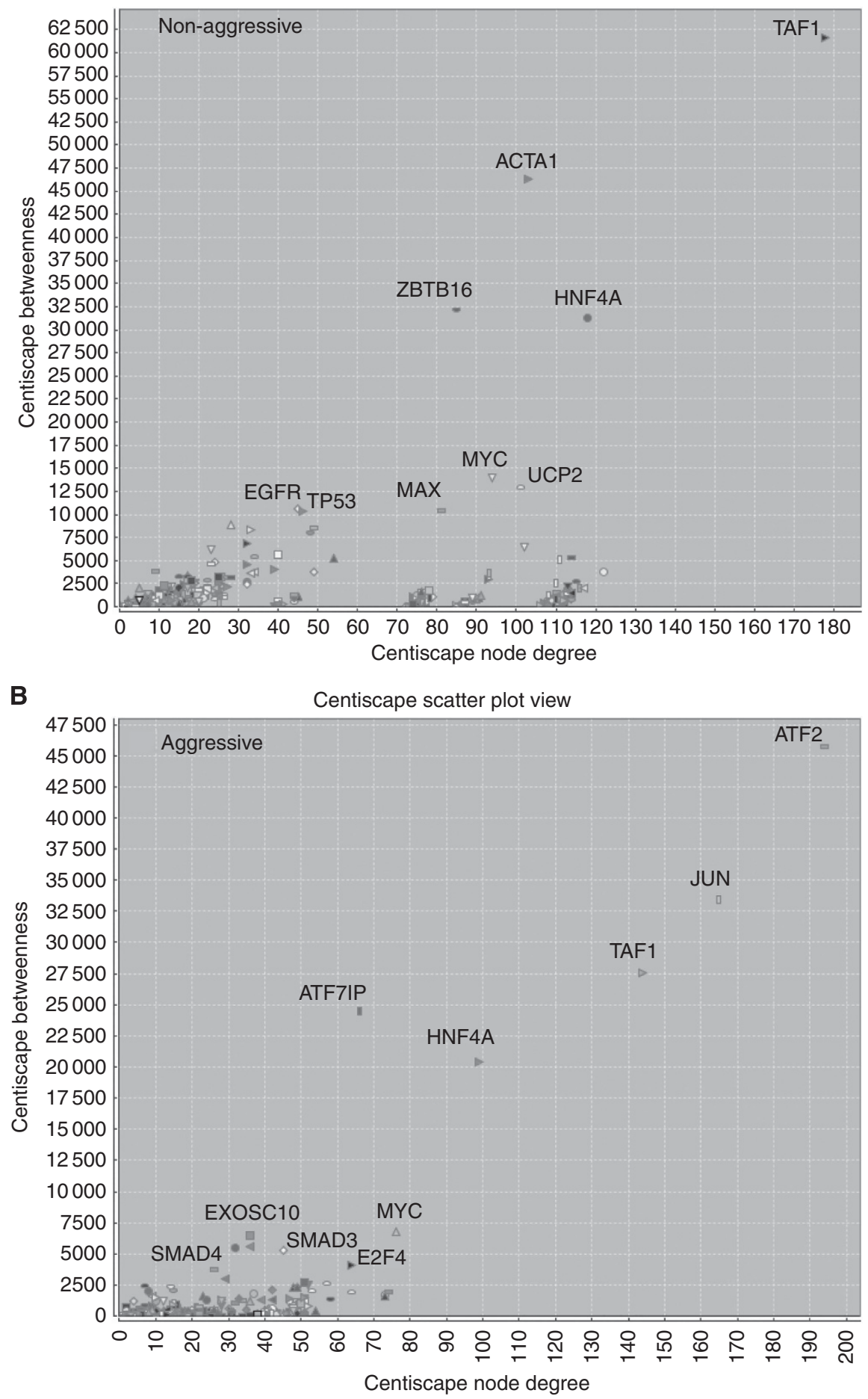

Figure 3. Network analysis of individualised proteome profiling identifies proteins central to affected molecular mechanisms. Betweenness centrality of nodes of (A) non-aggressive and (B) aggressive-associated networks is shown. The nodes with high betweenness centrality were extracted by analysis of topology of the networks. The nodes are plotted as functions of degree connectivity and beweenness. Some of the nodes are annotated.

microarrays. For PKN1, sensitivity was $98 \%$ and specificity was $40 \%$ for both tissue microarrays.

\section{DISCUSSION}

Variability in the molecular profiles of tumours is the main obstacle for efficient cancer treatment. Here, we explored an approach in which three EEC cases were subjected to full-scale proteomic profiling and systemic study, taking into consideration the individual features of the tumours. The results of individual proteome profiling were then used in a meta-analysis of the generated individual profiles. A similar approach has been used in the profiling of breast tumours, and showed significantly improved insights into the mechanisms governing tumorigenesis (Zakharchenko et al, 2011). Our validation study using 


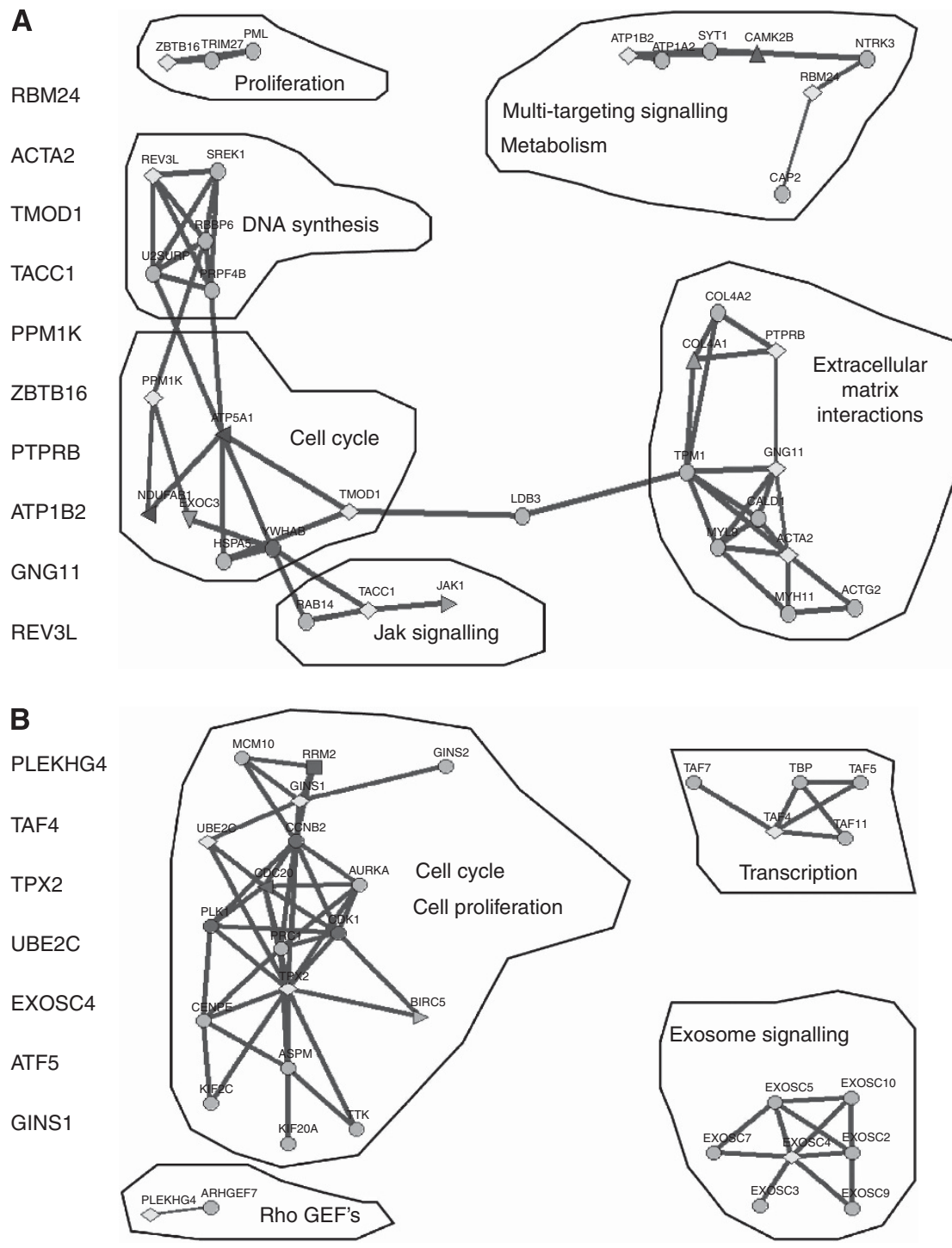

Figure 4. Combined meta-analysis of proteomics data and results of molecular profiling. Proteins and genes that were upregulated and correlated with non-aggressive (A) and aggressive (B) phenotypes of endometrial cancers are listed in the inserts of the panels. The proteins and genes are annotated in Gene Ontology terms. These proteins were used to build endometrial cancer-relevant networks using the FunCoup tool. Connections between proteins are set to a significance of $P<0.05$. Selected regulatory mechanisms represented in the networks are shown.

immunohistochemistry included a large cohort of patients (Figure 5) and confirmed the value of this approach for studying endometrial cancer.

Interpatient and intratumour variability in histological appearance, cellular composition, and molecular regulatory mechanisms have frequently been observed for many cancers, including endometrial (Saunders et al, 2012; Tian et al, 2012). This variability poses a serious hindrance to efficient cancer treatment. The high proportion of patients with partial response to treatment may be explained by the elimination of only a part of the tumour cells, while resistant cells then repopulate the tumour (Saunders et al, 2012). Molecular profiling of tumours has focused on studies of gene mutation and RNA expression (Kohlmann et al, 2012). Proteome profiling of endometrial tumours is increasing, and our report illustrates the positive impact of proteome studies on the improvement of diagnostic practices. Intact-protein proteomics by 2D gel electrophoresis coupled with mass spectrometry has been shown to be the most efficient way to explore full-length proteins, and therefore describe a true protein-based profile (Wilkins et al, 2006).
A combination of different OMIC studies have been instrumental in providing a much more comprehensive overview of the process than could be achieved using a single technology (Koboldt et al, 2012; Tian et al, 2012). The combination of proteomics, transcriptomics, genome sequencing, metabolomics, and clinical observations would be the best scenario for cancer diagnostics and treatment design. The combination of different techniques is also of importance to address issues of intertumour variations, such as variations in morphology. Such a combination ensures that the proteins identified in a proteomics study of a tumour would be validated by using sections of the tumour; and therefore, the origin of these proteins from malignant or other cells or stroma can be evaluated. Our results confirmed the usefulness of combining proteomics and transcriptomics, as this combination confirmed the relevance of our proteomics findings. However, lack of Protein Ontology and gaps in the representation of genes in transcriptomics studies create challenges due to missing values (Lan et al, 2003). Another challenge is identification of so-called 'novel' or 'unnamed' proteins, which were predicted by the genome and mRNA sequencing, but were not detected as proteins. As these 


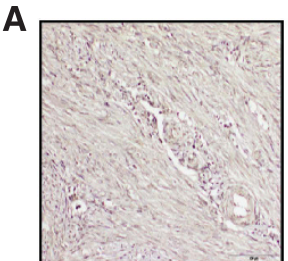

$(-)$

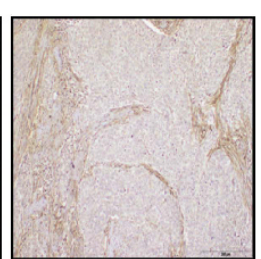

$(+)$

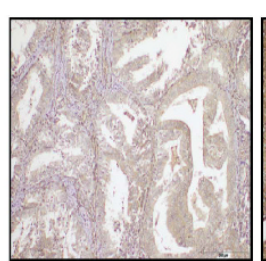

$(++)$

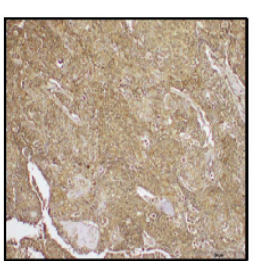

$(+++)$

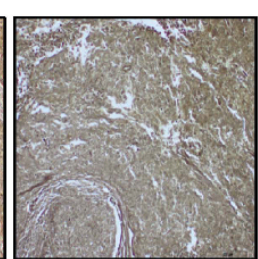

$(++++)$
B

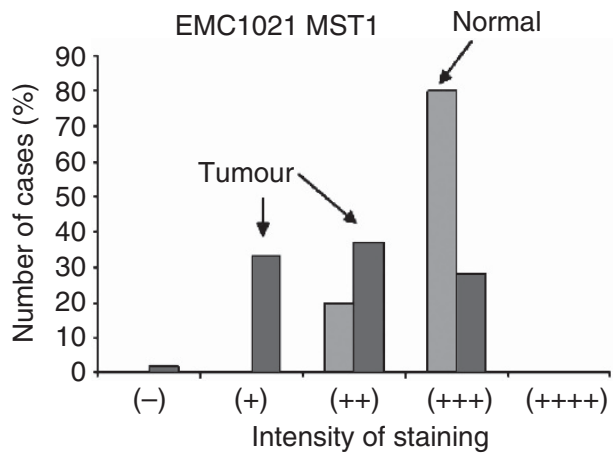

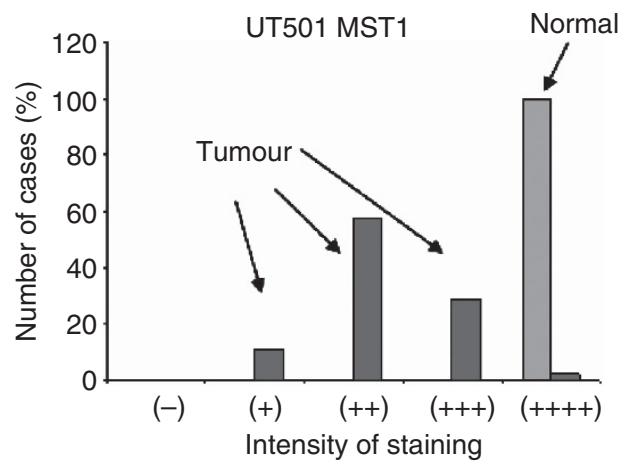

C

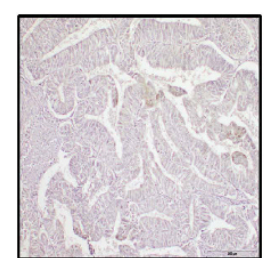

$(+)$

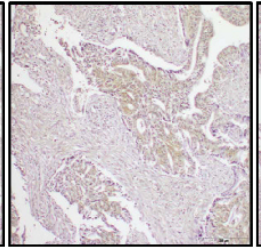

$(++)$

D

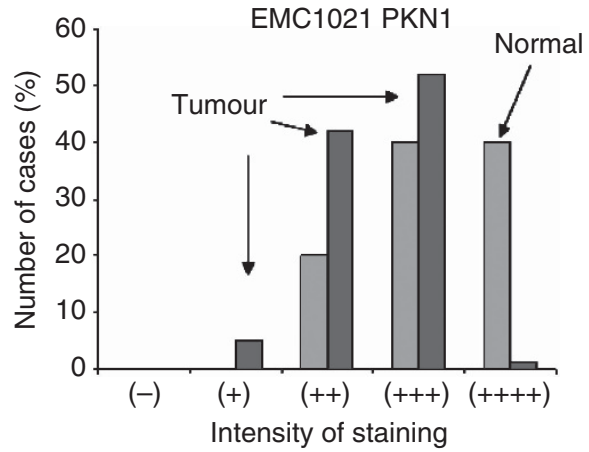

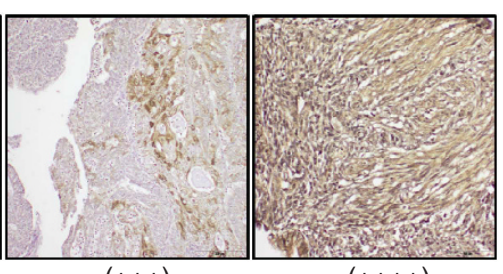

$(+++)$

$(++++)$

UT501 PKN1

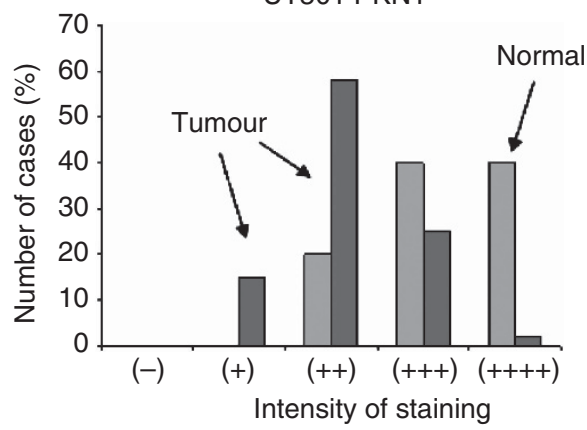

Figure 5. Expression of MST1 and PKN1 in the three EEC cases. Expression of MST1 (A and B) and PKN1 (C and D) in tumour and histologically normal adjacent tissues was monitored by immunohistochemistry. Brown colour indicates grading of staining for MST1 (A) and PKN1 (C). Representative images are shown (scale bars represents $200 \mu \mathrm{m}$ ). Quantification of staining of tumours and adjacent normal tissues is shown for MST1 (B) and PKN1 (D). The panels annotate which tissue microarrays were used, that is, EMC1021 and UT501.

proteins were not detected earlier, their functional role is not clear, and can be assumed only by homology to known proteins. These challenges are partially solved by systems biology tools, which allow for the exploration of dependencies between identified proteins and genes (Hucka et al, 2003). Building a network is the most frequent method employed to explore dependencies. It is also informative in the prediction of key regulators. The study of network topology allows for the identification of crucial functions and their regulators in a computer-assisted manner, which enhances the quality and significance of the findings (Hucka et al, 2003). Our Cytoscape-based study of the protein networks, and the combination of proteomics data and mRNA expression profiles led us to identify MST1 and PKN1 as potential regulators of endometrial tumorigenesis.

MST1 and PKN1 are kinases that have already shown their potential role in tumorigenesis. MST1 showed growth promoting activity in hepatocellular carcinoma cells upon NORE1B downregulation ( $\mathrm{Ng}$ et al, 2013), while loss of cytoplasmic MST1 expression has been reported to be a marker of tumour progression in colorectal cancer (Minoo et al, 2007). It has been reported that overexpression of PKN1 correlated with aggressive ovarian (Galgano et al, 2009), colorectal (Carter et al, 2004), and prostate cancers (Metzger et al, 2003). Other findings suggested that PKN1 has a role in the development of invasive phenotypes of breast (Adam et al, 2000) and gastric (Liu et al, 2009) cancer cells. Despite the recent advances in knowledge regarding the role of PKN1 and MST1 in tumorigenesis, the involvement of these proteins in the development and progression of endometrial cancer has been as of yet unexplored. Our data suggested that PKN1 and MST1 may be potential predictive biomarkers of endometrial cancer. Statistical significance of differences in expression of MST1 and PKN1 in malignant and normal cells, together with observed sensitivity and 
specificity values suggest they could be used in a clinical setting as predictive biomarkers.

The growing notion that there may be as many as 100 molecular profiles of cancer has stimulated the design of studies focused on the extraction of individual tumour features (Souchelnytskyi, 2005; Koboldt et al, 2012; Tian et al, 2012). Our approach is an example of such a study. Our data showed that full-scale individual profiling of tumours may unveil the possibility of targeting the tumour of a specific patient via a specific cancer drugs. Therefore, the approach we used may bring us one step closer to true personalisation of cancer treatment.

\section{ACKNOWLEDGEMENTS}

We are grateful to Ingrid Calone for comments on the manuscript; Sujata Lalit Kumar for help with collection of samples; Trudy Perdrix-Thoma for editorial help; and to 'Oves Minnesfond' for support and encouragement. This work was supported in part by grants from Swedish Cancer Research foundation, Swedish Research Council, Radiumhemmet Research Foundation, SI, Personalised Cancer Medicine Unit-KI, Erasmus program KI-UWM, EurocanPlatform to Serhiy Souchelnytskyi, and from the Stockholm County Council and Karolinska Institutet (ALF; \#510 222), and the Swedish Labor Market Insurance to Miriam Mints.

\section{CONFLICT OF INTEREST}

The authors declare no conflict of interest.

\section{REFERENCES}

Adam L, Vadlamudi R, Mandal M, Chernoff J, Kumar R (2000) Regulation of microfilament reorganization and invasiveness of breast cancer cells by kinase dead p21-activated kinase-1. J Biol Chem 275(16): 12041-12050.

Attarha S, Mints M, Andersson S, Souchelnytskyi S (2011) Endometrial cancer and application of proteomics. Exp Oncol 33: 174-177.

Bork P, Jensen LJ, von Mering C, Ramani A, Lee I, Marcotte EM (2004) Protein interaction networks from yeast to human. Curr Opin Struct Biol 14(3): 292-299.

Carter JH, Douglass LE, Deddens JA, Colligan BM, Bhatt TR, Pemberton JO, Konicek S, Hom J, Marshall M, Graff JR (2004) Pak-1 expression increases with progression of colorectal carcinomas to metastasis. Clin Cancer Res 10(10): 3448-3456.

Creasman WT (1993) Prognostic significance of hormone receptors in endometrial cancer. Cancer 71: 1467-1470.

Dedes KJ, Wetterskog D, Ashworth A, Kaye SB, Reis-Filho JS (2011) Emerging therapeutic targets in endometrial cancer. Nat Rev Clin Oncol 8: 261-271.

Doll A, Abal M, Rigau M, Monge M, Gonzalez M, Demajo S, Colás E, Llauradó M, Alazzouzi H, Planagumá J, Lohmann MA, Garcia J, Castellvi S, Ramon y Cajal J, Gil-Moreno A, Xercavins J, Alameda F, Reventós J (2008) Novel molecular profiles of endometrial cancer-new light through old windows. J Steroid Biochem Mol Biol 108: 221-229.

Engelsen IB, Stefansson I, Akslen LA, Salvesen HB (2006) Pathologic expression of $\mathrm{p} 53$ or $\mathrm{p} 16$ in preoperative curettage specimens identifies high-risk endometrial carcinomas. Am J Obstet Gynecol 195: 979-986.

Galgano MT, Conaway M, Spencer AM, Paschal BM, Frierson Jr HF (2009) PRK1 distribution in normal tissues and carcinomas: overexpression and activation in ovarian serous carcinoma. HumPathol 40(10): 1434-1440.

Hucka M, Finney A, Sauro HM, Bolouri H, Doyle JC, Kitano H, Arkin AP, Bornstein BJ, Bray D, Cornish-Bowden A, Cuellar AA, Dronov S, Gilles ED, Ginkel M, Gor V, Goryanin II, Hedley WJ, Hodgman TC, Hofmeyr JH, Hunter PJ, Juty NS, Kasberger JL, Kremling A, Kummer U, Le Novère N, Loew LM, Lucio D, Mendes P, Minch E, Mjolsness ED,
Nakayama Y, Nelson MR, Nielsen PF, Sakurada T, Schaff JC, Shapiro BE, Shimizu TS, Spence HD, Stelling J, Takahashi K, Tomita M, Wagner J, Wang J. SBML Forum (2003) The systems biology markup language (SBML): a medium for representation and exchange of biochemical network models. Bioinformatics 19(4): 524-531.

Kauppila AJ, Isotalo HE, Kivinen ST, Vihko RK (1986) Prediction of clinical outcome with estrogen and progestin receptor concentrations and their relationships to clinical and histopathological variables in endometrial cancer. Cancer Res 46: 5380-5384.

Koboldt D, Cancer Genome Atlas Network (2012) Comprehensive molecular portraits of human breast tumours. Nature 490(7418): 61-70.

Kohlmann A, Grossmann V, Haferlach T. (2012) Integration of nextgeneration sequencing into clinical practice: are we there yet? Semin Oncol 39(1): 26-36.

Lan N, Montelione GT, Gerstein M. (2003) Ontologies for proteomics: towards a systematic definition of structure and function that scales to the genome level. Curr Opin Chem Biol 7(1): 44-54.

Levan K, Partheen K, Osterberg L, Olsson B, Delle U, Eklind S, Horvath G (2010) Identification of a gene expression signature for survival prediction in type I endometrial carcinoma. Gene Expr 14(6): 361-370.

Liu F, Li X, Wang C, Cai X, Du Z, Xu H, Li F (2009) Downregulation of p21-activated kinase-1 inhibits the growth of gastric cancer cells involving cyclin B1. Int J Cancer 125(11): 2511-2519.

Metzger E, Müller JM, Ferrari S, Buettner R, Schüle R (2003) A novel inducible transactivation domain in the androgen receptor: implications for PRK in prostate cancer. EMBO J 22(2): 270-280.

Mhawech-Fauceglia P, Wang D, Kesterson J, Clark K, Monhollen L, Odunsi K, Lele S, Liu S (2010) Microarray analysis reveals distinct gene expression profiles among different tumor histology, stage and disease outcomes in endometrial adenocarcinoma. PLoS One 5(11): e15415.

Minoo P, Zlobec I, Baker K, Tornillo L, Terracciano L, Jass JR, Lugli A (2007) Prognostic significance of mammalian sterile20-like kinase 1 in colorectal cancer. Mod Pathol 20(3): 331-338.

Monge M, Colas E, Doll A, Gil-Moreno A, Castellvi J, Diaz B, Gonzalez M, Lopez-Lopez R, Xercavins J, Carreras R, Alameda F, Canals F, Gabrielli F, Reventos J, Abal M (2009) Proteomic approach to ETV5 during endometrial carcinoma invasion reveals a link to oxidative stress. Carcinogenesis 30(8): 1288-1297.

Ng YK, Lau WS, Lui VW, Cheng AS, Ng PK, Tsui SK, Cheung YS, Lai PB (2013) Full-length Mst1 exhibits growth promoting function in human hepatocellular carcinoma cells. FEBS Lett 587(5): 496-503.

Pradhan M, Abeler VM, Danielsen HE, Sandstad B, Tropé CG, Kristensen GB, Risberg B (2012) Prognostic importance of DNA ploidy and DNA index in stage I and II endometrioid adenocarcinoma of the endometrium. Ann Oncol 23: 1178-1178.

Salvesen HB, Carter SL, Mannelqvist M, Dutt A, Getz G, Stefansson IM, Raeder MB, Sos ML, Engelsen IB, Trovik J, Wik E, Greulich H, Bø TH, Jonassen I, Thomas RK, Zander T, Garraway LA, Oyan AM, Sellers WR, Kalland KH, Meyerson M, Akslen LA, Beroukhim R (2009) Integrated genomic profiling of endometrial carcinoma associates aggressive tumors with indicators of PI3kinase activation. Proc Natl Acad Sci USA 106(12): 4834-4839.

Salvesen HB, Haldorsen IS, Trovik J (2012) Markers for individualised therapy in endometrial carcinoma. Lancet Oncol 13(8): e353-e361.

Salvesen HB, Iversen OE, Akslen LA (1999) Prognostic significance of angiogenesis and $\mathrm{Ki}-67, \mathrm{p} 53$, and p21 expression: a population based endometrial carcinoma study. J Clin Oncol 17: 1382-1390.

Saunders NA, Simpson F, Thompson EW, Hill MM, Endo-Munoz L, Leggatt G, Minchin RF, Guminski A. (2012) Role of intratumoural heterogeneity in cancer drug resistance: molecular and clinical perspectives. EMBO Mol Med 4(8): 675-684.

Sharon D, Chen R, Snyder M (2010) Systems biology approaches to disease marker discovery. Dis Markers 28: 209-224.

Souchelnytskyi S (2005) Bridging proteomics and systems biology: what are the roads to be traveled? Proteomics 5(16): 4123-4137.

Staff AC, Trovik J, Eriksson AG, Wik E, Wollert KC, Kempf T, Salvesen HB (2011) Elevated plasma growth differentiation factor-15 correlates with lymph node metastases and poor survival in endometrial cancer. Clin Cancer Res 17: 4825-4833.

Susini T, Amunni G, Molino C, Carriero C, Rapi S, Branconi F, Marchionni M, Taddei G, Scarselli G (2007) Ten-year results of a 
prospective study on the prognostic role of ploidy in endometrial carcinoma: dNA aneuploidy identifies high-risk cases among the so-called 'low-risk' patients with well and moderately differentiated tumors. Cancer 109: 882-890.

Tian Q, Price ND, Hood L. (2012) Systems cancer medicine: towards realization of predictive, preventive, personalized and participatory (P4) medicine. J Intern Med 271(2): 111-121.

Westin SN, Broaddus RR (2012) Personalized therapy in endometrial cancer. Cancer Biol Ther 13(1): 1-13.

Wilkins MR, Appel RD, Van Eyk JE, Chung MC, Görg A, Hecker M, Huber LA, Langen H, Link AJ, Paik YK, Patterson SD, Pennington SR, Rabilloud T, Simpson RJ, Weiss W, Dunn MJ. (2006) Guidelines for the next 10 years of proteomics. Proteomics 6(1): 4-8.
Yi Z, Jingting C, Yu Z (2009) Proteomics reveals protein profile changes in cyclooxygenase-2 inhibitor-treated endometrial cancer cells. Int J Gynecol Cancer 19: 326-333.

Zakharchenko O, Greenwood C, Lewandowska A, Hellman U, Alldridge L, Souchelnytskyi S (2011) Meta-data analysis as a strategy to evaluate individual and common features of proteomic changes in breast cancer. Cancer Genomics Proteomics 8(1): 1-14.

This work is published under the standard license to publish agreement. After 12 months the work will become freely available and the license terms will switch to a Creative Commons AttributionNonCommercial-Share Alike 3.0 Unported License.

Supplementary Information accompanies this paper on British Journal of Cancer website (http://www.nature.com/bjc) 